\title{
Incorporating free-form features in aesthetic and engineering product design: State-of-the-art report
}

\author{
J.-P. Pernot ${ }^{\text {a,* }}$, B. Falcidieno ${ }^{\text {b }}$, F. Giannini ${ }^{\text {b }}$, J.-C. Léon ${ }^{\mathrm{c}}$ \\ a LSIS, UMR CNRS 6168, Aix-en-Provence, France \\ ${ }^{\mathrm{b}}$ IMATI-CNR, Genova, Italy \\ c G-SCOP Laboratory, FRE CNRS 3028, Grenoble, France
}

Keywords:

Free-form surfaces

Geometric modelling

Design by features

Shapes and semantics

Integrated design

\begin{abstract}
A B S T R A C T
The use of free-form shapes has become mainstream to design complex products that have to fulfil engineering requirements as well as aesthetic criteria. Even if today's CAD systems can easily represent free-form shapes by means of NURBS surfaces, their definition and modification still require a deep knowledge and a great skill in the manipulation of the underlying mathematical models. The implemented free-form shapes design operators are time consuming and do not enable fast modifications. To overcome these limits, some researches have been undertaken to try to adapt the feature concept, successfully adopted for the design of regular shapes, in the free-form domain. It gives rise to a set of free-form features modelling strategies. This paper gathers together the state-of-the-art of these advances. The various approaches are depicted and compared with respect to a very precise set of criteria expressing the needs in aesthetic and engineering designs. The limits and future trends are presented.
\end{abstract}

\section{Introduction}

The increase of the customers' requirement level and the growing competition of the worldwide market makes that today's commercial success of a product neither depends on its quality, on its cost nor even on its technical performances. In fact, in many industrial areas, the customers' choices are highly influenced by more subjective criteria such as aesthetics, which is essentially conveyed through the product shapes. For example, the emotions the free-form shapes of a car coachwork are able to convey can play a fundamental role in the success of a new car (Fig. 1a). The use of very complex shapes is also mandatory in engineering design where the needs are related to functional criteria. It can be noted that in most cases, the product geometry is defined by combination of regular and free-form shapes. For example, the aerodynamic shape of turbine blades, which determines the performance of aircraft engines, requires the use of free-form-surfaces (Fig. 1b). On the contrary, the support of the same turbine blade is often defined by analytic surfaces, e.g. planes, cylinders, used to answer basic functional requirements, e.g. prismatic or cylindrical joints. In parallel, the availability of new materials and the evolution of the

\footnotetext{
* Corresponding author.

E-mail address: jean-philippe.pernot@aix.ensam.fr (J.-P. Pernot).
}

manufacturing processes have permitted wider freedom in a shape definition process becoming more and more complex.

Even if today's CAD systems can easily represent free-form shapes by means of NURBS surfaces [22], their definition and modification still require a deep knowledge and great skills in the manipulation of the underlying mathematical models. The implemented free-form shapes design operators are time consuming and do not enable fast modifications. Thus, the global reactivity of the product design and manufacturing processes is affected, which represents a problem in competitive engineering. The feature concept seems to be a good solution to overcome these limits. It has been successfully adopted for the design of products whose shapes are defined by analytic surfaces, e.g. planes, cylinders. Form features are used to give a meaning to a set of faces that are not anymore manipulated separately but as a single entity of higher semantic level than the pure geometry, e.g. holes, slots or ribs. Integrated in the early design phases, they enable fast modifications through the access to the feature model thus allowing an easy evaluation of solution alternatives. They are also a good mean to associate the semantic information corresponding to the various tasks of the design process. Fig. 2 shows an example of product whose definition requires the use of regular as well as freeform shapes. The regular shapes are defined by form features whereas the free-form shapes are still defined as a set of free-form surfaces resulting from low-level modelling operations such as 

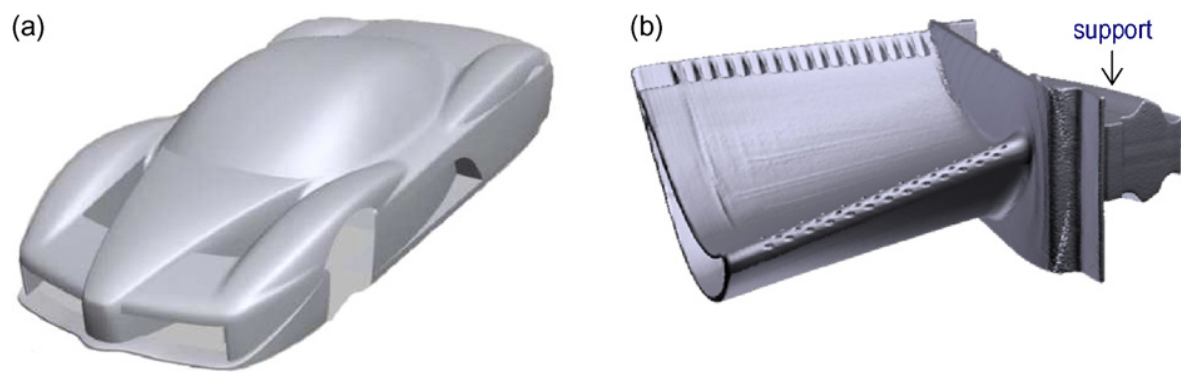

Fig. 1. The use of free-form shapes in aesthetic (a, courtesy Fiores project) and engineering designs (b, turbine blade made of regular and free-form shapes, courtesy Georgia Tech).

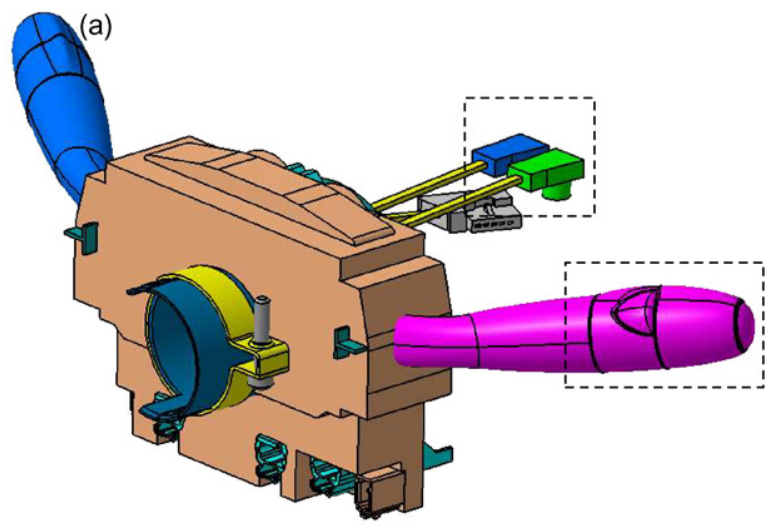

(b)

Fig. 2. (a) Geometric model of a commands bloc of a car. Commands bloc designed with (b) form features (analytic surfaces) and (c) free-form surfaces (courtesy PSA).

loft, sweep and so on. Sometimes, the designer must nevertheless adjust the shapes while moving the control points of the underlying NURBS surfaces. This is generally time consuming and requires a lot of expertise in knowing the corresponding effects on the surface. Several approaches have already been proposed to translate in the free-form domain the various concepts dedicated to form features. It gives rise to several free-form features modelling strategies that are widely studied and compared in this state-of-the-art report.

This paper is organized as follows. Section 2 shows the potential of feature-based modelling for Product Lifecycle Management. It also briefly depicts the main problems that have to be solved to transfer in the free-form domain the various concepts devoted to regular-shaped features. Section 3 introduces a very precise set of criteria used in Section 4 to compare the various approaches. These criteria express the needs in aesthetic and engineering designs. Finally, the limits and future trends are presented. They largely address the standardization of the shape feature concepts to the different phases of the design process.

\section{Feature-based modelling}

Being able to modify interactively the geometric models associated to a product is a necessary but not a sufficient condition to ensure the efficiency of the engineering design process. Indeed, during the design of a new product, the geometric model is subjected to several modification steps and this, at different stages of the design process. For example, the size of a stiffener defined by a stylist may not be compatible with the mechanical characteristics the engineers are interested in, e.g. the minimization of the maximum Von Mises stress. Therefore, the available tools should not only permit the definition of new shapes but also their modification through the use of high level operations such as copy/ paste, move, size changes, remove and so on. Furthermore, these modification tools have to be intuitive since different stakeholders of the design process can be interested in their use. In fact, to be able to meet these requirements, the modelling process should be shape oriented in the sense that the designer should think and build his/her geometric model through the use of high level entities and not directly through the manipulation of simple primitives (faces, edges). The stiffener the stylist and the engineer are sharing should be clearly delimited and identified as an entity taking part to the definition of the product shape. Such a way to proceed corresponds to the so-called feature-based modelling approaches.

The feature concept has been successfully adopted for the design of shapes defined by analytic surfaces such as planes, cylinders, spheres and so on [24]. Following the feature-based approach, the geometric model is not anymore perceived as a collection of vertices, edges and faces but as a well organized set of features corresponding to slots, ribs, stiffeners and so on. All the properties related to a feature type are specified within a feature class that defines a template for all its instances. This always includes the generic shape of the feature, and a number of parameters, e.g. length, width, and constraints, e.g. parallel, perpendicular, that characterize this shape. By specifying values for the parameters, an instance of the feature class can be created and then be added to a feature model [5]. The feature model usually contains the feature instances, the information related to their mutual dependencies and the chronology of construction.

Two approaches are classically adopted for the definition of a feature model: design by features and feature recognition. In design by features, the designer creates a feature model step by step while instantiating either predefined or user-defined features. Such an approach is already available in most of CAD software; it is based on the so-called B-Rep and C.S.G. modelling strategies. Fig. 3 depicts an example of such a strategy. Starting from the geometric model of a product, feature recognition techniques try to recognize 
(a)

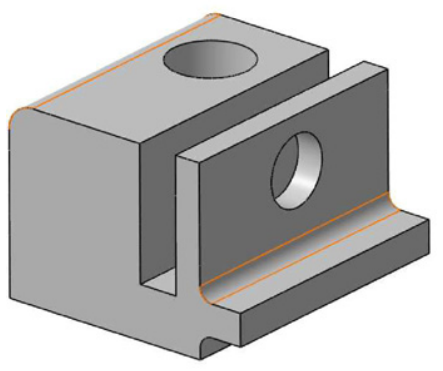

(b)

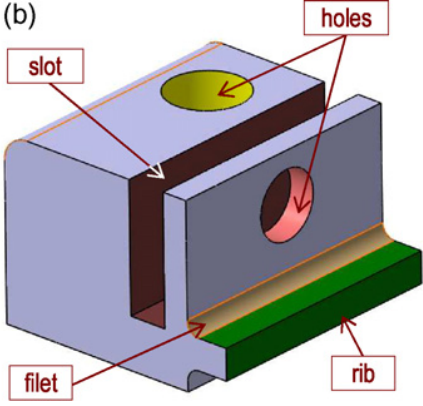

Fig. 3. An object designed with form features (b). A meaning is given to a set of geometric elements (faces, edges) which are not anymore considered separately (a) but as a single entity (hole, slot, rib, filet and so on).

or identify the features and to rebuild the feature model [24]. It has been also used to derive view-depend product descriptions, e.g. manufacturing.

Actually, even if the notion of feature is often associated to the geometric modelling aspects of the product design, it is not restricted to it. These high level entities may also enable the definition of a framework for the specification of additional information attached to the different tasks of the design process [24]. The simulation engineer can be interested in the definition of the materials attached to the different parts of the object. He/she can also be interested in the specification of boundary conditions directly expressed on the geometric elements, e.g. internal pressure inside a hole or prescribed displacements. Similarly, the manufacturing engineer can be interested in the specification of information related to its processes, e.g. the tolerances associated to each face or the type of tool used to manufacture the different parts. More generally, the features aim at conveying semantic information related to the different tasks of the product design process [3]. The treatment of these informations may need feature entities, i.e. face grouping, different from those meaningful for the design activity. Thus, suitable algorithms for their identification must be developed and their insertion in a unique feature model requires specific validity maintenance algorithms to check the validity conditions on, and between, the features, e.g. the manufacturability conditions [4]. Unfortunately, such a full integration of the tasks is not realistic. The provided solutions are still at the level of research prototypes $[6,14,15]$ and further investigations are required to transform actual feature modelling approaches into real multiple-view feature modelling systems for Product Lifecycle Management (PLM) [26]. Even if the final goal of the work proposed in this document is to reach this level of multirepresentations and multi-views of the product, this work mainly focuses on the geometric aspects of modelling by features. As a consequence, the state-of-the-art of the feature-based approaches will be restricted to the study of the geometric aspects of these high level entities.

\subsection{From regular-shaped features to free-form features}

As already stated in Section 1, in both aesthetic and engineering designs, the stylists/designers may be interested in the definition and manipulation of complex shapes defined by free-form surfaces. Even if parametric curves and surfaces such as Bézier, B-Spline or NURBS [22] have been successfully adopted for the design of such surfaces, their creation and modification still require a deep knowledge of the underlying mathematical models. It is mainly due to the fact that the manipulations are not shape oriented. To overcome these limits, the concept of form feature has been extended to free-form shapes thus giving rise to the so-called free-form features (FFF). In fact, most of the concepts addressed by form feature can be mapped to FFF $[17,30]$. This state-of-the-art report focuses on the so-called design by FFF approach. Fig. 4 shows two examples of such an approach for the insertion of complex shapes on a geometric model composed of several trimmed patches [19]. The different FFFs can be manipulated directly through a restricted set of high level control parameters introduced in Section 4.4.

In our opinion, to fully exploit the potential in product development both traditional analytical form feature and freeform features must be integrated within a single feature model, based on a multi-representation concept for the definition of geometric features independently of the underlying geometric representations, e.g. NURBS surfaces, meshes, subdivision surfaces [17].

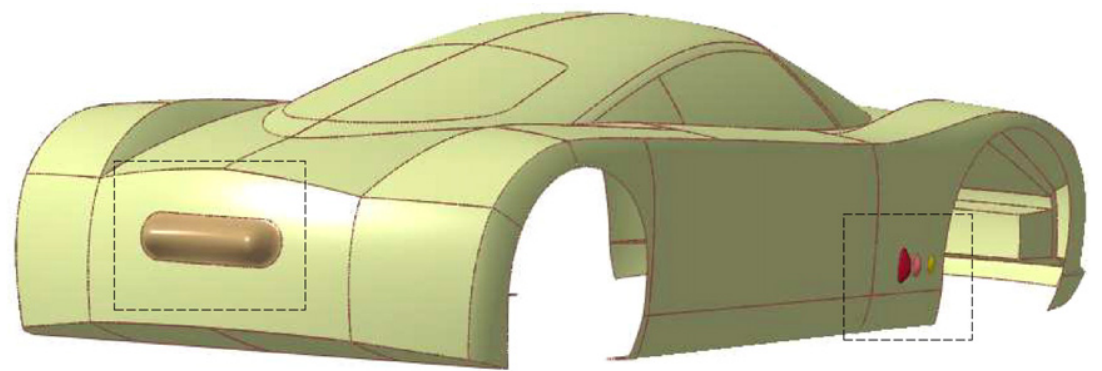

(a)

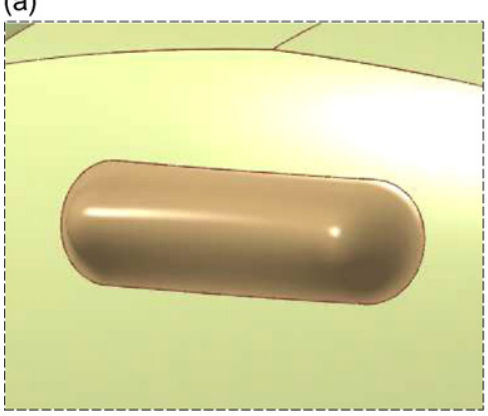

(b)

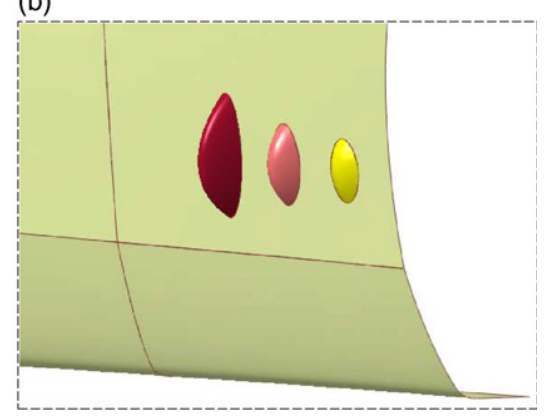

Fig. 4. Design by free-form features (FFF) [19]. (a) Bump free-form feature and (b) pattern of bumps. 


\section{Feature-based approaches evaluation criteria}

To carry out appropriate analyses and comparisons between the existing feature-based approaches, suitable evaluation criteria and an adapted ranking system have to be adopted. On one hand, the various criteria define the framework for the analyses. They depend on the application domain needs and characteristics. Considering the aesthetic and engineering design of styling products, four main categories of criteria have been identified (Sections 3.1-3.4): the criteria related to the adopted geometric models and features, those attached to the shapes and functions, those related to the families of shapes and those concentrating on the interactivity provided by the approaches. On the other hand, the ranking system enables a qualitative classification of the various approaches with respect to the specified criteria. Here, a Boolean scale is sufficient to characterize the capabilities of the approaches. The symbols $\ominus$ (respectively $\oplus$ ) are used to tag the methods not very adapted (respectively well adapted) with respect to the considered criterion (Table 1 ). They state a negative (more $\ominus$ ) or positive (more $\oplus$ ) tendency of the approaches with respect to the given criteria. They are defined in such a way that the optimal method would never be assigned the symbol $(\ominus)$. In case the information contained in the articles do not enable the assessment of a criterion, the symbol (?) is used. The symbol $(\odot)$ indicates criteria that have no meaning for the method. Of course, this synthesis results from our understanding of the analysed publications.

\subsection{Criteria related to geometric models and features}

The first criterion could have been relative to the type of feature manipulated, either volume or surface features. Since both can be interesting in aesthetic and engineering designs, this criterion has not been considered as a discriminatory one and it will not appear in the tables. In the styling domain, surface features seems better appropriate, since in several cases the resulting shape is not a volume but just a complex surface such as a car coachwork (Fig. 4). Volume features fit well the needs in mechanical engineering and notably for the definition of complex shapes such as the outer case of a hydraulic pump [27].

The two first selected criteria distinguish the methods applicable to parametric surfaces (one or several patches) or polyhedral models (structured or unstructured). The third criterion is used to differentiate the methods preserving the initial model from those modifying it (Table 2). Since semantic information may be associated to the elements, e.g. boundary conditions on points,

Table 1

Symbols used to characterize the approaches

\begin{tabular}{ll}
\hline Symbols & Criteria \\
\hline$\ominus$ & Not adapted \\
$\oplus$ & Well adapted \\
$?$ & Not appreciable \\
$\odot$ & No meaning \\
\hline
\end{tabular}

Table 2

Criteria related to geometric models and features

\begin{tabular}{llll}
\hline $\begin{array}{l}\text { Criteria relative to geo- } \\
\text { metric models and features }\end{array}$ & Gradation of criteria \\
\cline { 3 - 4 } & $\ominus$ & $\oplus$ \\
\hline a & Parametric surfaces & Single patch & Multiple patches \\
b & Polyhedral models & Structured & Unstructured \\
c & Preservation & Models are different & Models are identical \\
d & Discontinuities & Prescribed & Free \\
e & Dependencies & Dependent & Independent \\
\hline
\end{tabular}

curves, surfaces and so on, forming the geometric model, it is effectively important to try to preserve $(\mathbf{c} \oplus)$ these information while avoiding the modification (c $\ominus$ ) of the model topology, e.g. the number of patches and their connections, during the successive manipulation steps. Such capabilities can reduce the information losses when transferring the model to other modelling systems or when removing an entity or bad propagation of this information during the addition of new entities. Moreover, surface trimming brings problems in keeping the connectivity and continuity in the model, and the insertion of small surfaces may result in successive manufacturing difficulties. Nevertheless, for aesthetic reasons, it can be necessary to modify the topology for inserting discontinuities (position, tangency) in continuously derivable models, i.e. the parametric curves and surfaces in the present case. When these singularities can appear either along the boundaries of connected patches, or at the end points of curves, or along isoparametric directions, the discontinuities are said prescribed $(\mathrm{d} \ominus$ ). At the opposite, the singularities are defined as being free $(\mathrm{d} \oplus)$ when they can appear along any free-form curves lying on a set of connected trimmed patches.

The fifth criterion of this category enables the characterization of the dependencies to the underlying geometric models. To claim to be shape oriented, the definition of the features must be as much as possible independent $(\mathrm{e} \oplus$ ) of the underlying geometric model on which they are applied. Approaches that would use specific configurations of the surface control points, their decomposition into patches, for the definition of their features are not acceptable $(\mathrm{e} \ominus$ ).

\subsection{Criteria related to shapes and functions}

The first criterion indicates whether a classification of the FFF is proposed and, in this case, it characterizes the extent of the classification. To be pertinent, the classification must be exhaustive $(f \oplus)$. It should contain subclasses enabling an easy access to the different shapes the user can 1 day desire to access (Table 3 ).

As explained in [13], the styling activity is mainly a curve-based activity. To draw their shapes, the stylists first sketch the curves that define the character lines of the object to model. As a consequence, the definition of the shapes/features must be curve oriented in the sense that it should be possible to define complex shapes through the direct specification of a set of curves $(\mathrm{g} \oplus$ ) possibly coming from other applications, e.g. laser scanners or virtual reality haptic systems.

The parameterization of these shapes should also be possible $(\mathrm{h} \oplus$ ). Such a possibility is important in engineering design where the designers are mainly interested in the definition of shapes characterized by main dimensions, e.g. length, width, height or depth. Nevertheless, the number of parameters must be kept small $(\mathrm{i} \oplus)$ without however reducing the designers' creativity. Finally, in order to help the designers/stylists in the definition of the various parameters, explicit validity constraints should be accessible. Offering facilities to impose such conditions on features can be

Table 3

Criteria related to shapes and functions

\begin{tabular}{llll}
\hline \multicolumn{2}{l}{$\begin{array}{l}\text { Criteria related to shapes and func- } \\
\text { tions }\end{array}$} & Gradation of criteria & \\
\cline { 3 - 4 } & & $\ominus$ & $\oplus$ \\
\hline $\mathrm{f}$ & Classification & Partial & Exhaustive \\
$\mathrm{g}$ & Curve oriented & No & Yes \\
$\mathrm{h}$ & Parameterization & Impossible & Possible \\
$\mathrm{i}$ & Number of parameters & Important & Minimized \\
$\mathrm{j}$ & Validity constraints & Basic & Advanced \\
\hline
\end{tabular}


Table 4

Criteria related to families of shapes

\begin{tabular}{llll}
\hline \multicolumn{2}{l}{$\begin{array}{l}\text { Criteria related to families of } \\
\text { shape }\end{array}$} & Gradation of criteria & \\
\cline { 3 - 4 } & & $\ominus$ & $\oplus$ \\
\hline $\mathrm{k}$ & Local shape & Difficult & Easy \\
$\mathrm{l}$ & Global shape & Difficult & Easy \\
$\mathrm{m}$ & Level of freedom & Low & High \\
$\mathrm{n}$ & Diversity & One solution & Several solutions \\
$\mathrm{o}$ & Level of control & Homogeneous & Heterogeneous \\
\hline
\end{tabular}

very helpful in creating valid models only. Basic validity constraints (j॰) corresponds to quite easily manageable constraints such as "this length has to be positive" whereas more advanced validity constraints $(\mathbf{j} \oplus)$ such as the specification of Min/Max curvatures, Min/Max volume or the requirement for no self-intersections is much more difficult to handle.

\subsection{Criteria related to families of shapes}

A complete feature-based approach should enable the definition of both local and global shapes $(\mathrm{k} \oplus, \mathrm{l} \oplus$, Table 4). These two types of features reflect the needs arising during the two main steps of the design process, i.e. the overall shape definition followed by local refinements [13]. When defining local shapes, the level of freedom of the feature boundaries must be high $(\mathrm{m} \oplus)$. It should neither be restricted to parametric directions nor trimming curves $(\mathrm{m} \ominus$ ).

Once the main dimensions (width and so on) of a feature are defined, several solutions should be accessible $(n \oplus)$ and manageable by qualitative parameters such as "flat area" or "round area". These various behaviours should be applicable either homogeneously $(0 \ominus$ ), i.e. a unique type of behaviour can be used, or heterogeneously $(0 \oplus)$, i.e. several behaviours can be used on different areas. Such capabilities characterizes the level of control of the features. These two last criteria are key elements in the classification of the different feature-based approaches which is proposed in the next section. They distinguish the approaches that use wholeheartedly the power of description of NURBS surfaces from the others. Moreover, being able to characterize the various behaviours is a first step for the semantic enrichments.
Table 5

Criteria related to the interactivity of the approaches

\begin{tabular}{llll}
\hline $\begin{array}{l}\text { Criteria related to the } \\
\text { interactivity }\end{array}$ & Gradation of criteria \\
\cline { 3 - 4 } & $\ominus$ & $\oplus$ \\
\hline $\mathrm{p}$ & Manipulation & Tedious & Simple \\
$\mathrm{q}$ & Operators & One category & Several categories \\
\hline
\end{tabular}

\subsection{Criteria related to the interactivity of the feature-based approaches}

The first criterion evaluates the simplicity of the manipulations carried out by the user (Table 5). The greater the number of control parameters $(i \ominus)$, the more tedious and time consuming the manipulation is and the less interactive it is $(\mathrm{p} \ominus)$. The qualitative manipulations are also welcome in addition to the prescriptive ones, i.e. features defined by dimensions and so on. Several categories of operators $(q \oplus)$ should be available to enable the direct manipulation of the features as if they were made of clay. Such possibilities are particularly well adapted to the styling activity where a great freedom is required. It is also a first step toward the definition of a semantic-based system closer to the designers' intents.

\section{State-of-the-art report of FFF-based approaches}

As previously stated, this state-of-the-art report gathers together existing techniques for the definition and manipulation of free-form Features, whereas all the other techniques concerning the reverse engineering of FFF or the recognition of FFF on freeform surfaces have not been considered. These approaches can be characterized by the level of shape control of the resulting shapes. Based on this assumption, three main categories of FFF can be distinguished (Fig. 5):

- Semi free-form features defined by free-form surfaces, obtained by classical rules such as loft/sweep operations, interpolation rules or specific relationships directly expressed between the control points. The user often has few possibilities to control the resulting shapes.

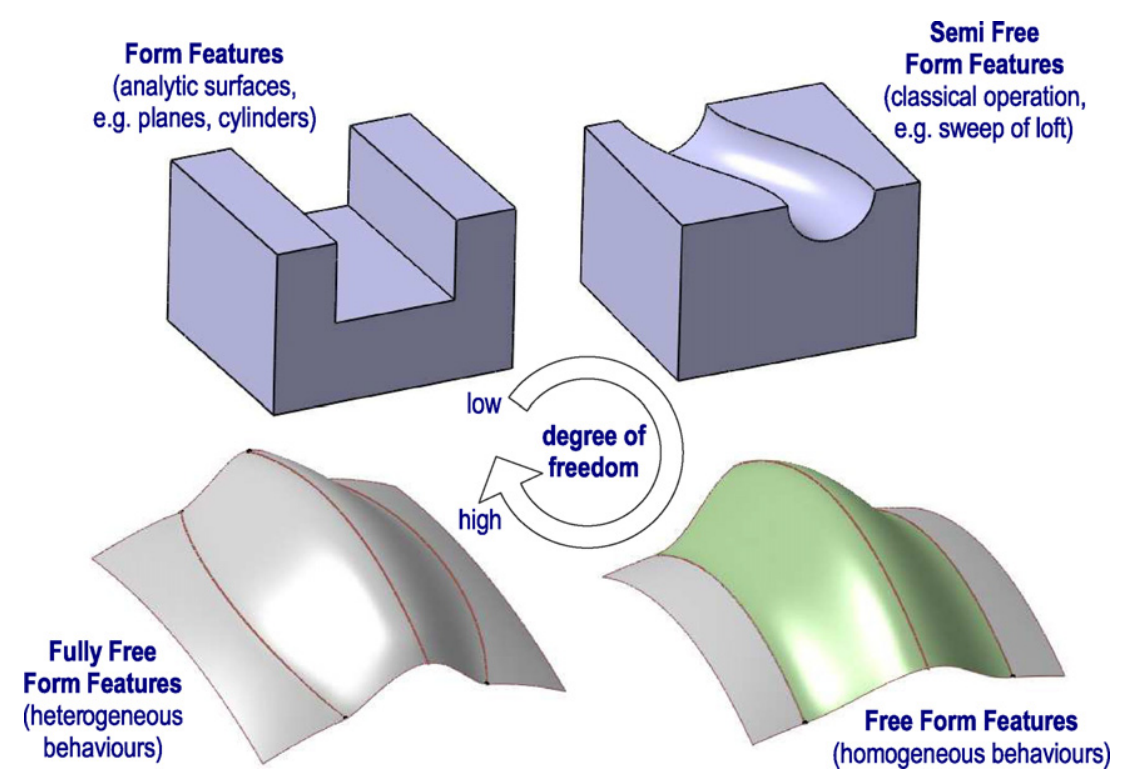

Fig. 5. Features classification based on the concept of level of control. 
Table 6

Feature-based approaches analysis

\begin{tabular}{|c|c|c|c|c|c|c|c|}
\hline \multirow[t]{3}{*}{ Criteria } & & \multicolumn{6}{|c|}{ Feature-based approaches } \\
\hline & & \multirow[t]{2}{*}{ Form features } & \multicolumn{3}{|c|}{ Semi FFF } & \multirow[t]{2}{*}{ FFF } & \multirow[t]{2}{*}{$\mathrm{F}^{4}$} \\
\hline & & & Point & Curve & Surface & & \\
\hline \multicolumn{8}{|c|}{ Geometric models and features } \\
\hline $\mathrm{a}$ & Parametric surfaces & $\oplus$ & $\ominus$ & $\oplus$ & $\ominus$ & $\oplus$ & $\oplus$ \\
\hline $\mathrm{b}$ & Polyhedral models & $\odot$ & $\odot \oplus$ & $\odot$ & $\odot$ & $\odot$ & $\ominus$ \\
\hline c & Preservation & $\ominus$ & $\ominus$ & $\ominus$ & $\ominus$ & $\ominus$ & $\oplus$ \\
\hline $\mathrm{d}$ & Discontinuities & $\ominus$ & $\odot$ & $\oplus$ & $\ominus$ & $\ominus$ & $\oplus$ \\
\hline $\mathrm{e}$ & Dependencies & $\theta$ & $\ominus$ & $\oplus$ & $\ominus$ & $\oplus$ & $\oplus$ \\
\hline \multicolumn{8}{|c|}{ Shapes and functions } \\
\hline f & Classification & $\ominus$ & $\ominus$ & $\oplus$ & $\ominus$ & $\oplus$ & $\oplus$ \\
\hline $\mathrm{g}$ & Curve oriented & $\ominus$ & $\ominus$ & $\oplus$ & $\oplus$ & $\oplus$ & $\oplus$ \\
\hline $\mathrm{h}$ & Parameterization & $\oplus$ & $\oplus$ & $\oplus$ & $\oplus$ & $\ominus$ & $\oplus$ \\
\hline $\mathrm{i}$ & Number of parameters & $\oplus$ & $\oplus$ & $\oplus$ & $\oplus$ & $\ominus$ & $\oplus$ \\
\hline $\mathrm{j}$ & Validity conditions & $\ominus$ & $\ominus$ & $\oplus$ & $\ominus$ & $\ominus$ & $\ominus$ \\
\hline \multicolumn{8}{|c|}{ Families of shape } \\
\hline $\mathrm{k}$ & Local shape & $\oplus$ & $\oplus$ & $\oplus$ & $\oplus$ & $\oplus$ & $\oplus$ \\
\hline 1 & Global shape & $\theta$ & $\ominus$ & $\ominus$ & $\ominus$ & $\ominus$ & $\theta$ \\
\hline $\mathrm{m}$ & Level of freedom & $\ominus$ & $\ominus$ & $\oplus$ & $\oplus$ & $\oplus$ & $\oplus$ \\
\hline $\mathrm{n}$ & Diversity & $\ominus$ & $\ominus$ & $\ominus$ & $\ominus$ & $\ominus$ & $\oplus$ \\
\hline o & Level of control & $\odot$ & $\odot$ & $\odot$ & $\odot$ & $\theta$ & $\oplus$ \\
\hline \multicolumn{8}{|c|}{ Interactivity of the approaches } \\
\hline $\mathrm{p}$ & Manipulation & $\oplus$ & $\ominus$ & $\oplus$ & $\ominus$ & $\ominus$ & $\oplus$ \\
\hline $\mathrm{q}$ & Operators & $\odot$ & $\odot$ & $\odot$ & $\odot$ & $\odot$ & $\oplus$ \\
\hline
\end{tabular}

(a)

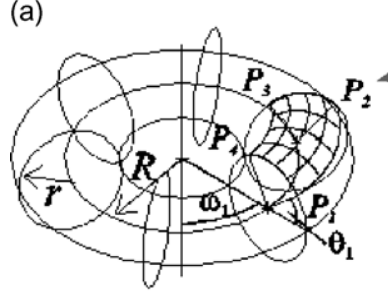

(b)

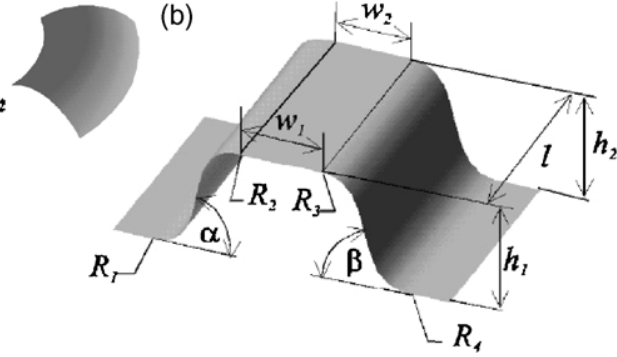

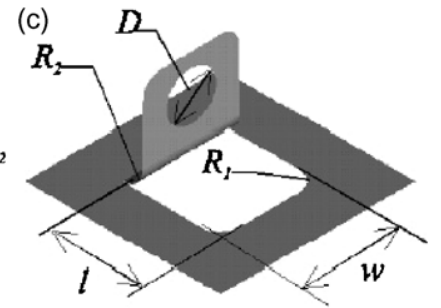

Fig. 6. Surface features coming from the main classes of the taxonomy proposed by Poldermann and Horvath [23]. (a) Primary surface (toroidal surface), modifying surface feature (lowered blend), (c) auxiliary surface feature.

- Free-form features defined by free-form surfaces, obtained through the use of adequate techniques expressing a homogeneous behaviour over the whole surfaces. There is more freedom but handling the modified area is not always as free as possible. The insertion of the features inside the initial model often requires topological changes, e.g. trimming operations, which is always risky (Section 3.1).

- Fully free-form features characterized by a higher level of freedom in the definition of the shapes obtained through the use of techniques prescribing heterogeneous behaviours over different areas of free-form surfaces. The modified area can be bounded by any free-form curve while preserving the surface initial topology (no trimming operations nor patches insertion). Such features take advantage of the NURBS description power [10] and fit well the stylists' requirements.

The results of the state-of-the-art report are collected in the final Table. In this study, all the feature-based approaches that do not use free-form surfaces have been considered as form features (Fig. 5). Section 4.1 gathers together the approaches which claim to be able to manage FFF whereas they simply deal with form features.

\subsection{Form features}

Poldermann and Horvàth [23] have introduced a design method based on the concept of surface features $(\mathrm{a} \oplus, \mathrm{b} \odot)$. In the proposed surface modelling system, the user can build its geometric model by using a set of parameterized surface features $(h \oplus, \mathrm{i} \oplus, \mathrm{j} \ominus$ ). A feature taxonomy is proposed. It contains four main classes:

- the primary surface features (PSF) represent the global characteristics of the surface. They are often used at the beginning of the design process and correspond to planes, conic surfaces, spherical surfaces, toroidal surfaces (Fig. 6a) and so on;

- the modifying surface features (MSF) are used to modify the primary shape locally. They can be wave, blend (Fig. 6b), protrusion, depression and so on;

- the auxiliary surface features (ASF) are similar to the MSF but have some mechanical functions. They can be holes, ears (Fig. 6c) and so forth;

- the transition surface features (TSF) are patches used to ensure appropriate smoothness at surface boundary connections $(\mathrm{d} \ominus$ ).

Fig. 7 shows the design of a stereo amplifier's front panel. On this example, the PSF is a simple plane. For a more complex example, the definition of the overall shape of the object from the set of simple PSF defined by the authors could be relatively difficult $(l \ominus)$. At the opposite, the local modifications could be relatively easy $(\mathrm{k} \oplus)$ even if their boundaries are quite simple $(\mathrm{m} \ominus)$. To each set of parameters corresponds a unique solution ( $\ominus$ ). No specific behaviour can be assigned $(0 \odot)$ and all the modifications require the modification of the surface topology $(\mathrm{d} \ominus$ ). 

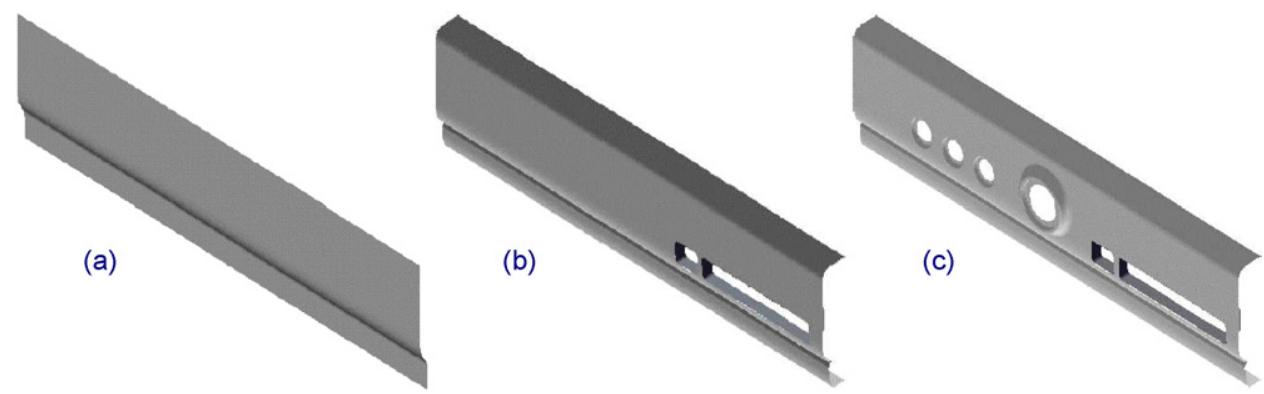

Fig. 7. (a-c) Three steps of the design process of a stereo amplifier's front panel [23].

This set of surface features does not really correspond to freeform shapes since they can be represented by implicit functions $(\mathrm{e} \ominus, \mathrm{q} \odot)$. In fact, most of the proposed features can be obtained by using classical functions available in most of the CAD software. However, the term "free-form" appears in certain sub-classes, but neither examples nor classification of these free-form entities are provided $(\mathrm{f} \ominus$ ). The manipulations are easy $(\mathrm{p} \oplus)$ but they are not curve-oriented $(\mathrm{g} \ominus$ ).

\subsection{Semi free-form features (semi FFF)}

This set of approaches can be decomposed into three main categories according to the type of constraints used to define the features. They distinguish point-based, curve-based and surfacebased semi FFF.

\subsubsection{Point-based semi FFF}

In his approach of the design by features, Vosniakos [34] defines the concept of free-form surface features on top of the Bézier geometric model $(\mathrm{b} \odot)$. More precisely, several untrimmed Bézier patches defined by $4 \times 4$ control points $(a \ominus)$ are used as "a standard acceptable choice" to represent the set of features defining an object (Fig. 8a). The example of Fig. 8b shows the geometric model decomposition of a telephone handset into three features: the earpiece, the mouthpiece and the body.

The boundary of each feature is defined by the boundaries of its constitutive patches $(\mathrm{m} \ominus$ ). The features are controlled by high level parameters $(\mathrm{h} \oplus)$ specified by the user and acting directly on the control points of the patches $(e \ominus$ ). In the proposed example, the earpiece is controlled by its global position, width, elongation, roundness and two different depths $(\mathrm{i} \oplus, \mathrm{j} \ominus$ ). No curve constraints can be used $(g \ominus)$. The tangency continuity between the different patches $(\mathrm{d} \odot)$ is ensured by additional constraints linking directly $(\mathrm{e} \ominus$ ) the control points of the adjacent patches (Fig. 8b). Once all these constraints specified, the set of control points is fully defined $(n \ominus, 0 \odot)$. This property justifies the term of semi FFF.

Even if this approach seems to be quite simple for the manipulation of the telephone handset, the manipulation of much more complex free-form shapes from a simple set of $4 \times 4$ untrimmed Bézier patches seems compromised $(p \ominus)$. Effectively, what about defining another product or slightly modifying the telephone by incorporating a new feature $(c \ominus, \mathrm{k} \ominus, \mathrm{l} \ominus)$ ? In addition, a classification based on an adjacency property is briefly proposed $(\mathrm{f} \ominus)$. It distinguishes touching, non-touching and connecting features but no more information are given about its use.

Vergeest and Horvàth [33] have proposed a complex formalism for the parameterization of free-form features. This formalism has been applied to the definition of a structure for the parameters of free-form ridges and holes $(\mathrm{f} \ominus, \mathrm{g} \ominus$ ). Thus, it mainly corresponds to the definition of local shapes $(\mathrm{k} \oplus, \mathrm{l} \ominus)$. In their approach, a ridge is defined by six parameters $(i \oplus, j \ominus)$ as shown in Fig. 9. The parameters of most interest to the user are the three "control points" $c_{1}, c_{2}$ and $c_{3}$, the width of the ridge $w$ and its height $h$. The parameter $a$ defines the extent of the single patch defining the feature. Similarly to the approach of Vosniakos, these high level parameters are used to define the position of the control points
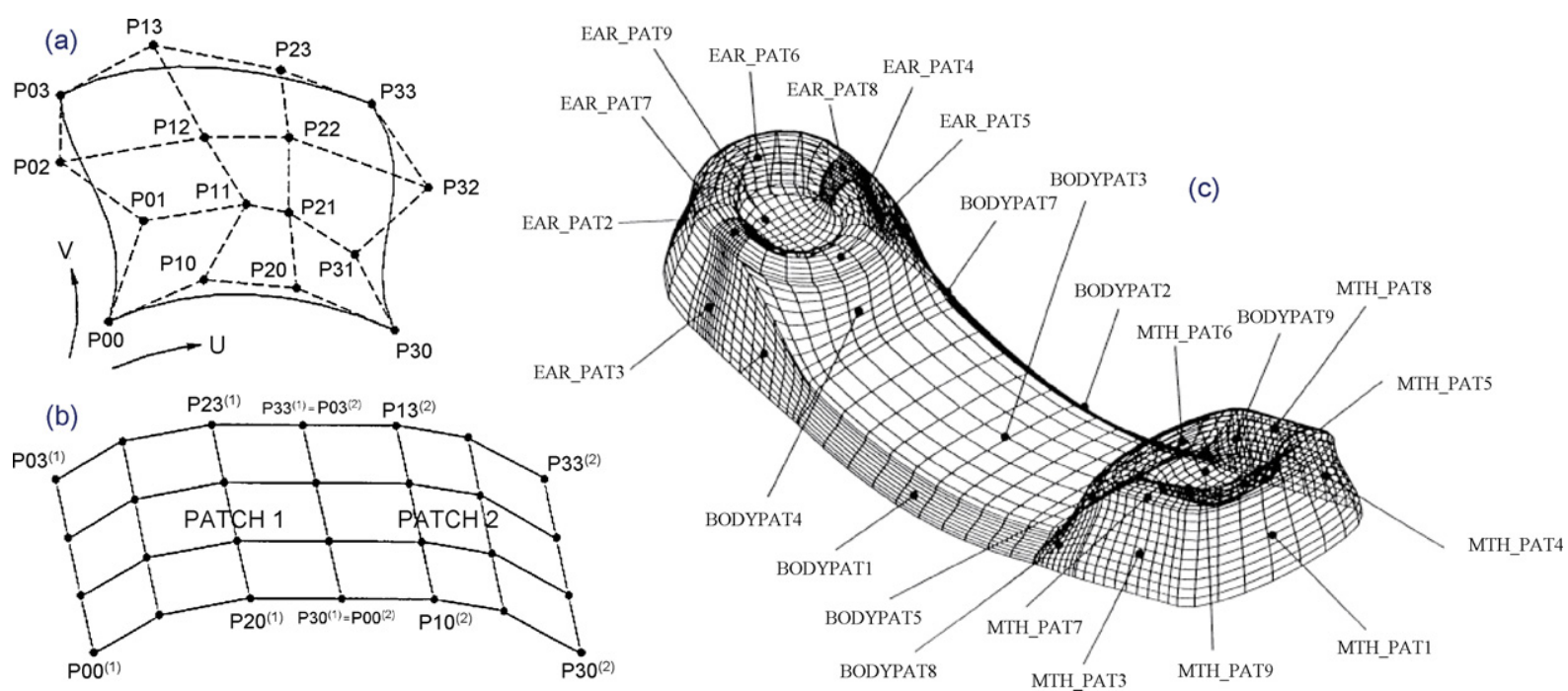

Fig. 8. A $4 \times 4$ Bézier patch (a) as basis for the definition of the features composing a telephone handset (c). The control points position is directly governed by higher level parameters, e.g. width and roundness, and continuity conditions between adjacent patches (b). Images from [34]. 


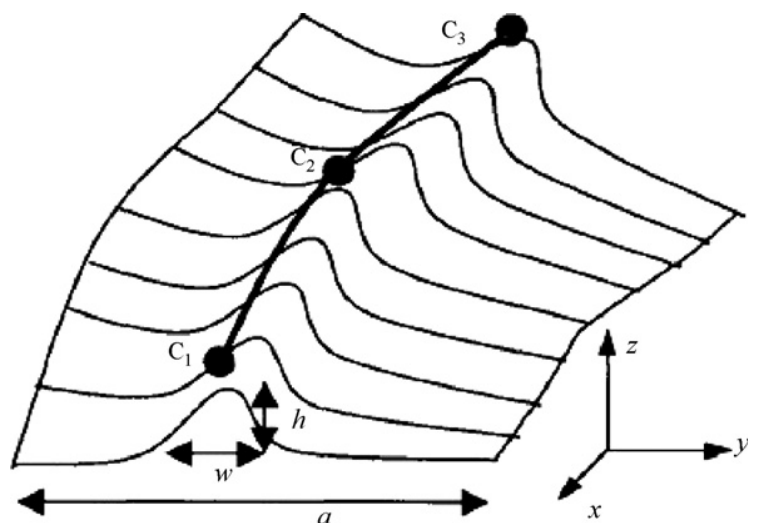

Fig. 9. Free-form ridge parameterization [33].

(e $\ominus$ ) of the surface representing the feature. More precisely, the surface control points are obtained by sweeping a row of control points (defined by $w, h$ and $a$ ) along a path (defined by the three points $c_{i}$ ). Once these parameters are defined, the surface is fully described $(\mathrm{n} \ominus, \mathrm{o} \odot)$. As a result, the use of only three points $c_{i}$ does not add to this approach a real curve-oriented capability $(g \ominus)$. Such a modelling process justifies the semi FFF designation together with the point-based categorisation. The boundary of the ridge directly corresponds to the boundary of the untrimmed patch $(a \ominus$, $\mathrm{m} \ominus$ ). The insertion of such elements inside an initial geometric model may require several adjustment steps, e.g. trimming operations $(\mathrm{c} \ominus$ ).

This definition of a free-form ridge has also been used in a reverse engineering context. Starting from a cloud of points of a scanned ridge, the software application determines the value of the numerical parameters $\left(c_{i}, h, w\right.$ and $\left.a\right)$ that minimize the Hausdorff distance. Using another sweeping scheme, it should be possible to extend this concept of free-form ridge to polyhedral models $(b \oplus)$. Unfortunately, the set of accessible shapes is very restricted.

In their recent works, Song et al. [25] have incorporated the concept of feature template to define free-form bumps, ridges and holes. A feature template is a parameterized free-form shape. Unfortunately, this approach still use high level parameters ( $w, h$ and so on) to fully define the location of the surface control points $(\mathrm{e} \ominus$ ) according to predefined rules $(\mathrm{o} \odot)$. The approach is not yet curve-oriented $(\mathrm{g} \ominus$ ), even for the definition of complex ridge (Fig. 10).
Still in the category of point-based semi FFF, Au and Yuen [2] have been working on the definition of a semantic language for modelling sculptured objects, i.e. objects made of free-form shapes. In fact, it is a formalization of what Vosniakos was able to do. They define the vocabulary and the corresponding grammar to build the object and apply it to model a mannequin (f $\ominus$, Fig. 10(a)). In their example, the features correspond to the different parts of the mannequin (neck, shoulder, chest and so on). They are defined by Bézier patches connected together with continuity conditions (Fig. 10(b)). The control points of the patches are obtained by a linear combination of some characteristic points $\mathbf{q}_{i}$ which are the only control parameters $(\mathrm{h} \oplus, \mathrm{i} \oplus, \mathrm{j} \ominus$ ) offered to the user. Once all these parameters specified, the mannequin is fully defined $(n \ominus, o \odot)$. This property justifies the point-based semi FFF categorisation. Here again, the notion of feature clearly depends on the patch decomposition which is too restrictive for free-form modelling (e $\ominus$ ).

One can notice that none of the proposed approaches enables the manipulation of the surfaces with interactive operators $(q \odot)$. All these manipulations are then prescriptive.

\subsubsection{Curve-based semi FFF}

In the approach of $[27,31]$, features are defined by means of a shape prototype. To each feature class corresponds a shape prototype. The prototypes are built from a set of the so-called free-form feature definition points (FFDP) that can be located in the 3D space. These points are key elements in the definition of the curves characterizing the feature. Fig. 11a shows the shape prototype of a curved rib feature. Eight points $\mathrm{p}_{i}$ are mandatory to define both the section curve and the path of the sweep operation defining this feature class. In order to ease the definition of the different points, parameters such as width, height, depth or shear can be defined (Fig. $11 \mathrm{~b}, \mathrm{~h} \oplus, \mathrm{i} \oplus$ ). Once a shape prototype is defined, its parameters can be instantiated. The new position of the FFDP are computed, thus defining new curves as input of the sweep operation.

In their approach, many shape prototypes can be theoretically defined by the user [29]: it can be a free-form extrusion, a free-form wrap or a blend $(\mathrm{f} \oplus)$. Fig. 11c illustrates the through hole feature class which is a sub-class of the free-form extrusion feature class. All of them use classical sweep operation $(e \oplus)$ and the shapes are therefore fully characterized $(\mathrm{n} \ominus, \mathrm{o} \odot$ ) by the different curves $(\mathrm{g} \oplus$ ), themselves defined by parameters constraining the FFDP. This property justifies the curve-based semi FFF categorisation. Even if

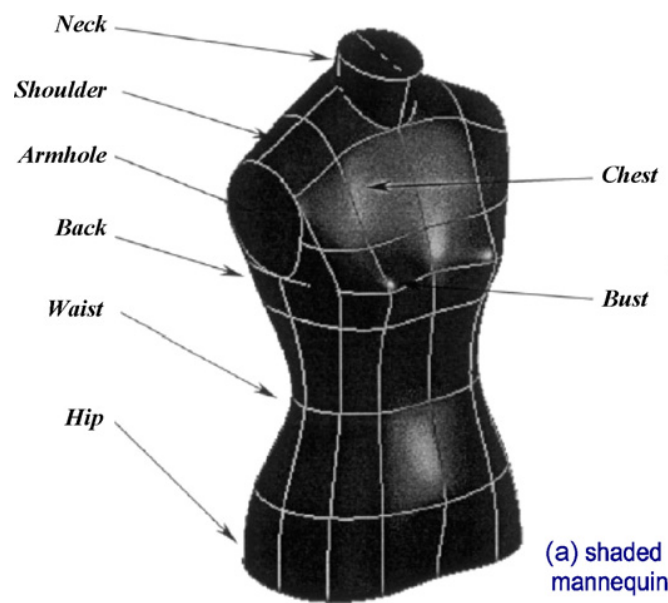

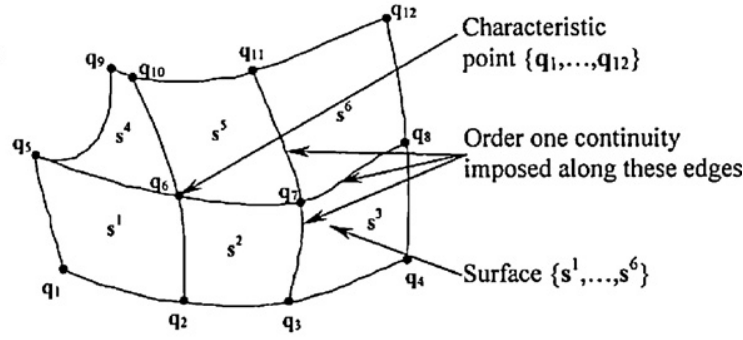

(b) bust feature defined by 6 surfaces 

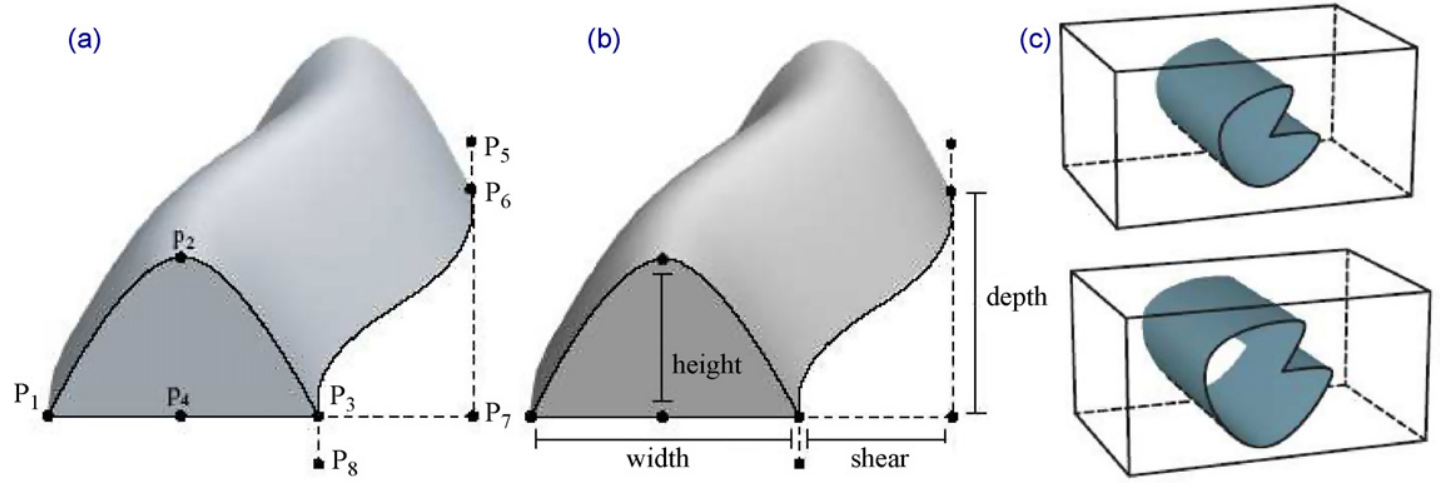

Fig. 11. Construction of a shape prototype from eight FFDP (a). Definition of higher level parameters (b). Examples of through hole features (c). Images from [31].

according to us, volumetric features are less appropriate in the styling domain, this type of features finds very interesting applications in engineering design, where the needs for defining complex shapes with a great level of freedom are important $(a \oplus$ $\mathrm{b} \odot)$. These classes incorporate local modifications $(\mathrm{k} \oplus, \mathrm{l} \ominus$ ) whose areas are bounded by free-form curves $(\mathrm{m} \oplus)$. The insertion of such features into an existing model is realised through the definition of attachments $(\mathrm{p} \oplus,[28])$. The topology of the initial model is modified (c $\ominus$ ) and, depending on the shape of the curves, discontinuities can appear $(\mathrm{d} \oplus)$. In their approach, not only the constraints can be specified, but also the validity conditions/ constraints can be expressed to ensure the validity of the resulting shapes $(\mathbf{j} \oplus)$.

Recently, a similar approach has been presented [16] for the definition of free-form surface features classes. The surface results from the interpolation (lofting or skinning) of curves themselves defined by interpolation ( $\ominus$ ) of FFDP parameterized and constrained by higher level parameters such as height, width and so on. Reading the paper, it is not clear how the features are applied on an initial geometric model, e.g. topological changes $(\mathrm{c} \ominus)$, and how the method can handle multiple trimmed patches which is widespread in mechanical engineering.

\subsubsection{Surface-based semi FFF}

Cavendish and Marin [8] have proposed a feature-based

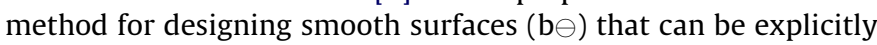
described as the graph of a single-valued bivariate function $z=$ $f(x, y)(\mathrm{e} \ominus)$. Such surfaces often occur in practice in manufacturing involving stamping or injection moulding operations. The design process starts with a primary surface $S_{0}$ on which a curve $\mathcal{C}_{0}$ has been drawn to locate the influence area $\Omega_{0}$ of the local modification ( $\mathrm{g} \oplus, \mathrm{k} \oplus, \mathrm{l} \ominus, \mathrm{m} \oplus$ ). The feature to be inserted in this area is defined by integrating a secondary surface $S_{1}$, bounded by a closed curve $\mathcal{C}_{1}$, with a transition surface such that $S_{1}$ smoothly joins $S_{0}$ between $\mathcal{C}_{1}$ and $\mathcal{C}_{0}$ (Fig. 12a). The definition of the transition surface is widely explained in their paper. It should ensure position as well as tangency continuities between the various surfaces. No control parameter is accessible to the user $(\mathrm{h} \ominus, \mathrm{i} \ominus, \mathrm{j} \ominus, \mathrm{n} \ominus, \mathrm{o} \odot)$ and the transition surface is computed automatically which justifies the semi FFF categorisation. No classification is proposed and the only accessible shapes are the protrusions and pockets $(\mathrm{f} \ominus$ ).

Later, Cavendish [7] has combined this feature-based method with the free-form deformation (FFD) techniques. The features are inserted at a preliminary step. Then, the surface incorporating all the features is immersed inside a FFD block whose modification creates the surface global deformation. Thus, shapes that cannot be simply represented as a function $z=f(x, y)$ are accessible.

Unfortunately, the surfaces manipulated by Cavendish do not correspond to NURBS surfaces $(a \ominus$ ) and additional translation phases have to be performed $(\mathrm{c} \ominus$ ) to integrate this method in a design process. Using such an approach, the complexity of the feature definition is transferred to the design of the secondary surface $(p \ominus)$. At the end, the initial problem is still unsolved since a surface still has to be designed by the user! In fact, this approach is useful for applications where surfaces are rather planar.

van Elsas and Vergeest [32] have used a similar method to define their displacement features. The curves $\mathcal{C}_{i}$ and the surfaces $S_{i}$ are assumed to be B-Spline $(e \oplus)$. In their approach, the secondary surface $S_{2}$ is obtained by conversion of the primary surface $S_{1}$. The definition of a new feature is simplified since only the boundary curves, the height of the displacement and two rounding parameters $R_{1}$ and $R_{2}$ are specified by the user (h $\oplus, \mathrm{i} \oplus, \mathrm{j} \ominus$, Fig. 12b). Such an approach can be interesting for some specific applications but it cannot be considered as a general FFF-based approach.

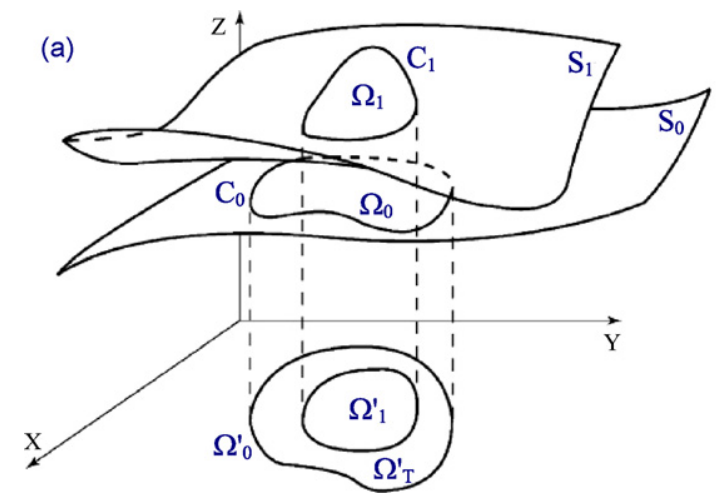

(b)

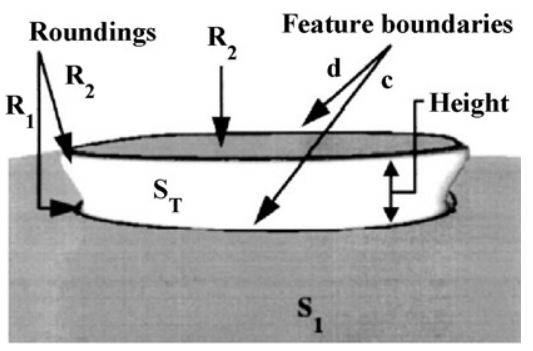

Fig. 12. Outline of feature boundary curves on primary and secondary surfaces (a, [8]). Particular case defining a displacement feature (b, [32]). 


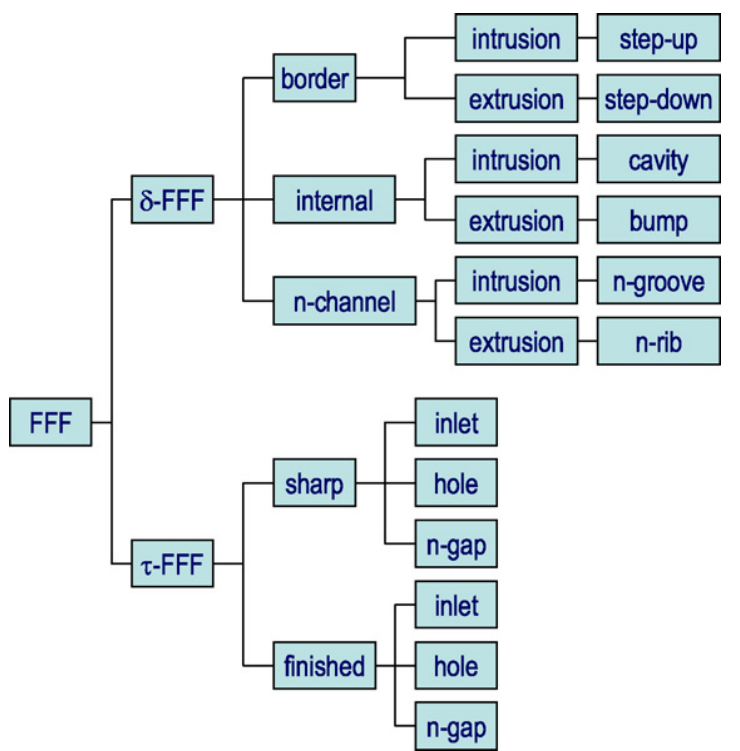

Fig. 13. FFF classification of [12].

\subsection{Free-form features (FFF)}

Fontana et al. [13] have proposed an interesting study of the concept of free-form features in the context of aesthetic design. Starting from an analysis of the styling activity, it appears that the definition of a shape is performed according to two logical steps: the product overall shape definition followed by local refinements. Moreover, the styling activity is a curve-based activity. The stylists express their ideas when drawing of a set of characteristic curves defining the shapes. In order to fasten the creation and modification of the shapes during these phases, two categories of FFF have been identified:

- the structural features which are created in the preliminary phase of design. They represent the structural entities used for defining the main surfaces $(a \oplus, b \ominus)$ of a product thus having an important aesthetic impact;

- the detail features which are created in the second modelling phase. They are applied to a surface in order to add aesthetic and functional details and to enforce the visual effects of important shape elements.

In $[12,13]$, the authors mainly focus on the specification, the classification and the implementation of detail features modifying

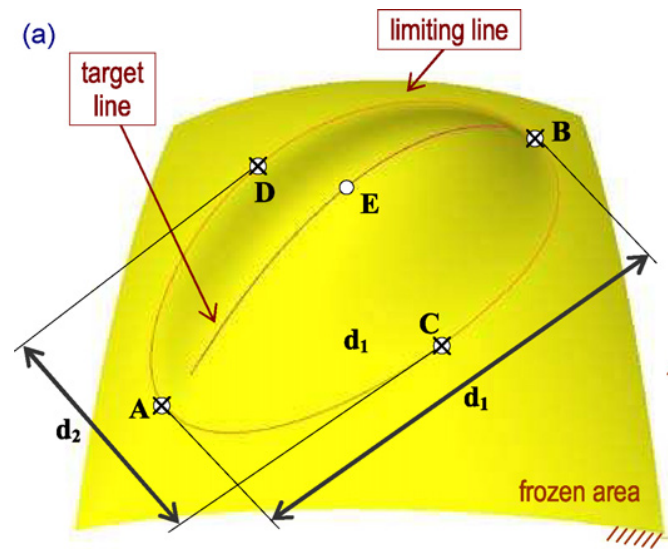

a local area of a surface $(\mathrm{k} \oplus, \mathrm{l} \ominus)$. This type of feature can be related to either an addition, or a substitution, or a removal operation. The first type of feature corresponds to the classical fillets or blending features that the authors have not considered since they are already well-known. The two other types respectively correspond to features obtained by deformation ( $\delta$-FFF) and features obtained by elimination ( $\tau$-FFF).

The deformation features are then classified according to the morphological and topological properties of the shapes. The morphological property distinguishes the intrusions from the extrusions whereas the topological property distinguishes the border, the internal and the channel features. The different eliminations are classified according to the finishing operation, i.e. either a sharp or a finished cut (e $\oplus$, Fig. 13).

In their implementation of the deformation features, the authors use the FdF deformation function developed by MATRA DATAVISION within the Brite-Euram project FIORES [11]. This technique generates a homogeneous behaviour of the surface ( $e \oplus$, $\mathrm{o} \odot)$. All the parameters of the deformation technique become control parameters enabling the access to a wider variety of shapes $(\mathrm{n} \oplus$ ). In the proposed example, the deformation area is bounded by two lines defined along isoparametric directions $(\mathrm{m} \oplus)$. These curves are used to trim the surface $(c \ominus)$. A handle line is used to constrain the surface to pass through it $(\mathrm{g} \oplus)$. Unfortunately, no real parameterization is proposed $(\mathrm{h} \ominus, \mathrm{i} \ominus, \mathrm{j} \ominus$ ). The treatment of discontinuities has been proposed but it is not really controlled within the prototype software $(\mathrm{d} \ominus$ ). The use of a deformation technique enables a greater level of freedom in the shape manipulation which justify the FFF categorisation.

\subsection{Fully free-form features $\left(F^{4}\right)$}

To overcome some limits of the approach proposed by Fontana et al. [13], the concept of fully free-form deformation features ( $\delta$ $\mathrm{F}^{4}$ ) as well as the associated creation/manipulation methods and tools have been introduced [19]. A $\delta-\mathrm{F}^{4}$ corresponds to an area of a free-form surface $(\mathrm{a} \oplus, \mathrm{b} \ominus, \mathrm{k} \oplus, \mathrm{l} \ominus)$ that results from a deformation (e $\oplus$ ) under specific constraint lines $(\mathrm{g} \oplus, \mathrm{m} \oplus)$ : the target, the limiting and driving lines. The target lines give the global directions of the shape whereas the limiting lines are free-form curves used to give a shape around the target lines and bound the deformation area (Fig. 14a). In this approach, the shape is inserted without modifying the surface topology $(\mathrm{c} \oplus$ ). It uses an automatic fixation algorithm to block the control points that have an influence outside the deformation area [17]. Driving lines correspond to curves used to drive the repetition of more simple shapes (Fig. 14b). A restricted set of high-level parameters are used

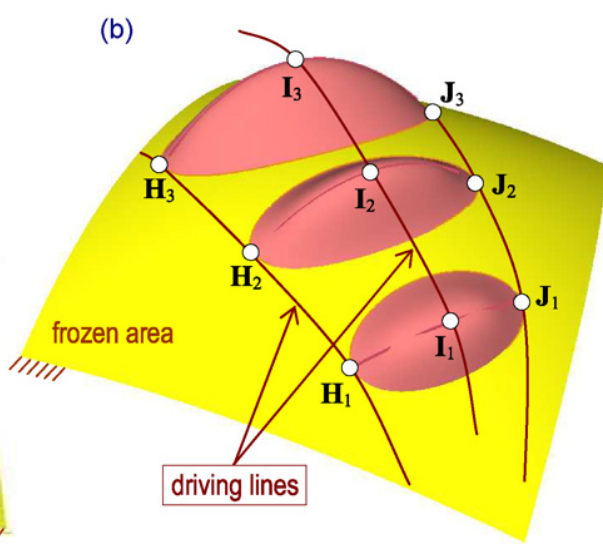

Fig. 14. A basic shape feature (a) defined by target and limiting lines parameterized with few numerical parameters and heterogeneous deformation behaviours. A complex shape feature defined as a repetition of BSF along several driving lines (b). 
to control both the shape of the curves and the shape of the surface around these curves $(i \oplus$ ). There are the numerical parameters (e.g. position, dimensions and orientation), the shape control parameters (type of multi-minimizations) and the type of discontinuity $\left(G^{0}, G^{1}\right)$. The numerical parameters are used to parameterize the features $(\mathrm{h} \oplus)$, the multiple minimizations enable the specification of heterogeneous $(n \oplus, 0 \oplus)$ shape behaviours [20] and the discontinuities can be inserted along any free-form directions $(\mathrm{d} \oplus)$. Additionally shape constraints, such as internal planar areas within a specific feature can be included, Cheutet [9]. The various parameters used to control the constraint lines can be instantiated in two ways using the concept of feature template: either the designers directly specify their own constraint lines or they can instantiate numerical parameters (dimensions, angles, shape behaviours and so on) used to shape the constraint lines with the generic deformation engine. Once defined over the surface, $\delta$ $\mathrm{F}^{4}$ can be manipulated in two ways $(\mathrm{p} \oplus)$ : either in a prescriptive manner while modifying the different parameters that have been instantiated (dimensions, shape behaviours and so on) or in a qualitative manner while using a set of interactive deformation operators, e.g. push, pull, $q \oplus$, Pernot et al. [21]. To select the $\delta-\mathrm{F}^{4}$ to be instantiated, a complete feature classification [18] has been proposed $(\mathbf{f} \oplus)$.

The strength of the proposed approach lies in the perfect harmony between degree of flexibility and shape control. The concept of $\delta-\mathrm{F}^{4}$ template enables mastered manipulations of the free-form curves that are imposed as constraints for the free-form surface deformation process. This double level of deformation (curve deformation followed by surface deformation) enables an easy access to a wide variety of shapes without limiting the designers' creativity. Moreover, the use of multi-minimizations initiates a new level of shape control thus justifying even more the term of fully free-form deformation features in comparison to the other approaches dealing with semi free-form features.

The main drawback of this approach lies in the fact that solely some basic validity conditions $(\mathrm{j} \ominus$ ) can be defined and managed through a rudimentary checker. Even if some works, e.g. [16,27], go deeper in the management of more advanced validity conditions $(\mathrm{j} \oplus)$, keeping all sorts of constraints valid is still an open issue. For example, the management of over-constrained configurations when deforming free-form surfaces has not been fully addressed yet.

\section{Conclusion and future trends}

This paper gathers together a state-of-the-art of the researches undertaken in the field of free-form feature modelling. The needs have been presented and the proposed approaches have been analysed and compared using a very precise set of criteria. Even if some aspects have already been successfully addressed, this research area is still open. The proposed tools are not yet sufficient to answer the growing needs of the industry which has now understood that aesthetics plays a major role in the success of a product.

More precisely, to be really adapted to the way the products should be designed using Product Lifecycle Management systems, several issues should be addressed:

- since several types of geometric models, e.g. surfaces, meshes are used all along the design process, the proposed approaches should enable the manipulation of FFF on these various models $(\mathrm{a} \oplus, \mathrm{b} \oplus)$ as well as on possibly hybrid ones, to avoid time consuming modification and conversion cycles. This corresponds to the multi-representation and multi-resolution issues;

- the features should enable local as well as global shape modifications $(\mathrm{k} \oplus, \mathrm{l} \oplus)$. This refers to the so-called detail and structural features introduced by [12];
- integration of the form features and FFF concepts to enable the definition of a product through a unique feature model (Fig. 2);

- ease the creation and maintenance of valid models through the specification and treatment of validity conditions [27] when deforming free-form surfaces $(\mathrm{j} \oplus$ );

- move towards semantic-based system for both defining the shapes of the product and associating information relative to the multiple views of the product (design, simulation and so on). See [9] for a recent survey in this field.

These issues are recognized internationally as they are being part of the research topics of the AIM@SHAPE European Network of Excellence [1].

\section{References}

[1] Aim@Shape, Advanced and innovative models and tools for the development of semantic-based systems for handling, acquiring, and processing knowledge embedded in multi-dimensional digital objects. European Network of Excellence, Key Action: 2.3.1.7 Semantic-based Knowledge Systems, VI Framework, 2004, URL: http://www.aimatshape.net.

[2] C.K. Au, M.M.F. Yuen, A semantic feature language for sculptured object modelling, Computer-Aided Design 32 (1) (2000) 63-74.

[3] R. Bidarra, W.F. Bronsvoort, Semantic feature modelling, Computer-Aided Design 32 (3) (2000) 201-225.

[4] R. Bidarra, K.J. de Kraker, W.F. Bronsvoort, Representation and management of feature information in a cellular model, Computer-Aided Design 30 (4) (1998) 301-313.

[5] W.F. Bronsvoort, R. Bidarra, P. Nyirenda, Developments in feature modelling Computer-Aided Design \& Applications 3 (5) (2006) 655-664.

[6] W.F. Bronsvoort, A. Noort, Multiple-view feature modelling for integral product development, Computer-Aided Design 36 (10) (2004) 929-946

[7] J.C. Cavendish, Integrating feature-based surface design with freeform deformation, Computer-Aided Design 27 (9) (1995) 703-711.

[8] J.C. Cavendish, S.P. Marin, A procedural feature-based approach for designing functional surfaces, in: H. Hagen (Ed.), Topics in Surface Modelling SIAM, Geometric Design Publications, Philadelphia, 1992, pp. 145-168.

[9] V. Cheutet, Towards semantic modelling of free-form mechanical products, Ph.D. Thesis, INP-Grenoble/University of Genoa, France/Italy, 2006.

[10] G.E. Farin, Curves and Surfaces for Computer-Aided Geometric Design: A Practical Guide, 4th edition, Academic Press, San Diego, 1996.

[11] Fiores, Formalization and integration of an optimized reverse engineering styling workflow. Brite-Euram Project N. BE96-3576, 1997-99, 1997, URL: http:// www fiores com.

[12] M. Fontana, F. Giannini, F. Meirana, A free form features taxonomy, Eurographics'99 18 (3) (1999) 107-118

[13] M. Fontana, F. Giannini, F. Meirana, Free form features for aesthetic design, International Journal of Shape Modelling 6 (2) (2000) 273-302.

[14] C.M. Hoffmann, R. Joan-Arinyo, Distributed maintenance of multiple product views, Computer-Aided Design 32 (7) (2000) 421-431.

[15] T. De Martino, B. Falcidieno, F. Giannini, Integrated feature-based modelling in concurrent engineering, in: TMCE'98 International Symposium on Tools and Methods for Concurrent Engineering, 1998, 381-392.

[16] P.J. Nyirenda, M. Mulbagal, W.F. Bronsvoort, Definition of freeform surface feature class, Computer-Aided Design \& Applications 3 (5) (2006) 665-674.

[17] J.-P. Pernot, Fully free form deformation features for aesthetic and engineering designs, Ph.D. Thesis, INP-Grenoble/University of Genoa, France/Italy, 2004, www.lsis.org/ jean-philippe_pernot.html.

[18] J.-P. Pernot, B. Falcidieno, F. Giannini, S. Guillet, J.-C. Léon, Modelling free form surfaces using a feature-based approach, in: Proceedings of the 8th ACM Symposium on Solid Modelling and Applications, Seattle, USA, (2003), pp. 270-273.

[19] J.-P. Pernot, B. Falcidieno, F. Giannini, J-C. Léon, Fully free-form deformation features for aesthetic shape design, Journal of Engineering Design (JED) 16 (2) (2005) 115-133.

[20] J.-P. Pernot, S. Guillet, J.-C. Léon, B. Falcidieno, F. Giannini, Shape tuning in fully free-form deformation features, Journal of Computing and Information Science in Engineering (JCISE) 5 (1) (2005) 95-103.

[21] J.-P. Pernot, S. Guillet, J.-C. Léon, B., Falcidieno, F. Giannini, Interactive operators for free form features manipulation, in: Geometric Modelling and Computing: Seattle 2003. Modern Methods in Mathematics, Selected Papers of the 8th SIAM Conference on Geometric Design and Computing, November 9-13, 2003, Nashboro Press, Brentwood, TN, 2004, pp. 437-452.

[22] L. Piegl, W. Tiller, The NURBS Book, Springer, New-York, 1995.

[23] B. Poldermann, I. Horvàth, Surface design based on parameterized surface features, in: Symposium on Tools and Method for Concurrent Engineering (TMCE'95), 1995, 432-446.

[24] J.J. Shah, M. Mäntylä, Parametric and Feature-based CAD/CAM, Wiley-Interscience Publication, John Wiley Sons, Inc., 1995.

[25] Y. Song, J.S.M. Vergeest, I. Horvàth, Reconstruction free-form surface with parameterized features, in: ASME, DETC'02, New-York, 2002. 
[26] J. Stark, Product Lifecycle Management-21st Century Paradigm for Product Realisation, Springer-Verlag, London, 2005.

[27] E. van den Berg, Freeform feature modelling and validity maintenance, Ph.D. Thesis, Delft University of Technology, 2007, http://graphics.tudelft.nl/Geometric_and_Feature_Modelling/RecentPublications.

[28] E. van den Berg, R. Bidarra, W.F. Bronsvoort, Construction of freeform feature models with attachments, in: CD-ROM Proceedings of the ASME DETC-CIE Conference, 2004.

[29] E. van den Berg, W.F. Bronsvoort, Parametrised, constraint-based wrapping of freeform shapes, Computers \& Graphics 31 (1) (2007) 89-99.

[30] E. van den Berg, W.F. Bronsvoort, J.S.M. Vergeest, Freeform feature modelling: concepts and prospects, Computers in Industry 49 (2) (2002) 217-233.

[31] E. van den Berg, H.A. van der Meiden, W.F. Bronsvoort, Specification of freeform features, in: ACM Symposium on Solid Modelling and Applications, Seattle, USA, (2003), pp. 56-64.

[32] P.A. van Elsas, J.S.M. Vergeest, Displacement feature modelling for conceptual design, Computer-Aided Design 30 (1) (1998) 19-27.

[33] J.S.M. Vergeest, I. Horvàth, Parameterization of freeform features, in: Proceedings of the IEEE Shape Modelling International Conference, 2001, pp. 20-28.

[34] G. Vosniakos, Investigation of feature-based shape modelling for mechanical parts with free form surfaces, International Journal of Advanced Manufacturing Technology 15 (3) (1999) 188-199.

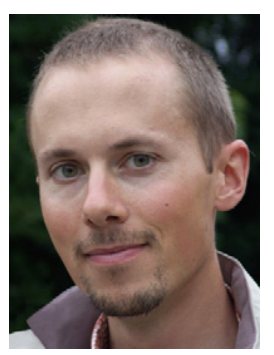

Jean-Philippe PERNOT is associate professor at the Ecole Nationale Supérieure des Arts et Métiers, Aix-enProvence, France. He received his $\mathrm{PhD}$ degrees in mechanical engineering from both the Institut National Polytechnique of Grenoble (INPG, France) and the Università degli Studi di Genova (Italy) in 2004. His $\mathrm{PhD}$ has been awarded by the INPG in 2005. His main research interests include geometric modelling, free form feature modelling, Finite Element mesh modification, polyhedral simplification and use of images for geometric model processing.

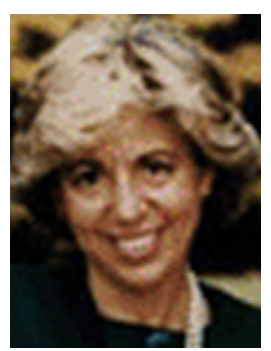

Bianca Falcidieno is a Research Director of the National Research Council (CNR) of Italy and the chair of the Department of Genova of the CNR National Institute of Applied Mathematics and Information Technology (CNR IMATI-GE). She has been leading and coordinating research at international level in advanced and interdisciplinary fields (such as computational mathematics, computer graphics, multidimensional media and knowledge technologies), strongly interacting with outstanding industrial and social application fields: from industrial design to geographic information systems, from manufacturing to semantic web. She is presently taking part in over ten European and Italian research projects and is the coordinator of the FP6 Network of Excellence AIM@SHAPE, aiming at representing and processing knowledge related to multi-dimensional media. From March 2008, she is the coordinator of the FP7 Coordination Action FOCUS K3D, whose main aim is to promote the adoption of best practices for 3D content knowledge modelling and processing. She is the author of over 200 scientific refereed papers and books, chair or co-chair of international conferences and workshops, and currently editor in chief of the International Journal Shape Modelling.

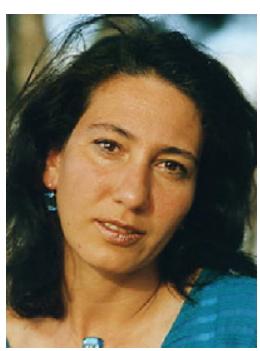

Franca Giannini is a senior researcher at the Institute for Applied Mathematics and Information Technologies of the National Council of Research (CNR) in Genova, Italy. She graduated in applied mathematics from the University in Genoa in November 1986. Since then she has concentrated on different issues for the specification of tools and methodologies for 3D geometric model representation, analysis and synthesis. In particular she focused on the development of shape processing and modelling tools adaptable to the application needs in industrial product development. She has participated and been responsible for IMATI in several national and international projects. The results of her research activity have been published in more than 90 reviewed papers. Her current research interests include multidimensional media modelling and understanding and related knowledge formalisation in applications contexts.

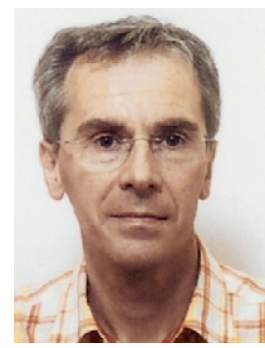

Jean-Claude Léon is Professor at GIT (Grenoble Institute of Technology), Mechanical Engineering faculty, vice-director of this faculty during nine years, he performs his research at the Laboratory G-SCOP and he is a member of the European network of excellence AIM@SHAPE. He received his PhD in mechanical engineering and automation from the Ecole Centrale Paris in 1985. The research area addressed cover the design of methods, algorithms, software tools aiding at the design of industrial products based on the concepts of digital mock-up, rapid prototyping, concurrent and collaborative engineering. More precisely, his research activities focus on the CAD/FEA link, the multiple representations of the product during the design phase (manufacturing model, assembly model, simulation models, ...). A strong know-how has been gained in the field geometric modelling, mesh generation and transformation, free-form surfaces deformation and variational geometry, technological information incorporated in geometric models. 